\title{
Memory and judgment: Availability versus explanation-based accounts
}

\author{
MICHAEL B.W. WOLFE and NANCY PENNINGTON \\ University of Colorado, Boulder, Colorado
}

\begin{abstract}
Two theories about the relationship between memory and decisions are examined. In an explanationbased model, the organization in memory of decision-relevant information determines decisions. In an availability model, the amount of information sampled from memory that supports competing decisions is crucial. In Experiment 1, subjects read evidence from a legal trial, made decisions, and provided free-recall data. Evidence presentation order was manipulated to produce differences in evidence coherence. When the prosecution evidence order was coherent, the subjects were more likely to choose guilty verdicts, and recall of prosecution evidence, relative to defense evidence, was greater. Thus, both evidence coherence and availability of information in memory could account for the basic findings. In Experiment 2, recall differences were eliminated as a function of evidence order. The results show that verdict effects favoring the more coherent prosecution evidence are obtained even when the relative amounts of prosecution and defense recall are equated across conditions.
\end{abstract}

One area of interest in decision-making research is the role that memory plays in the decision process. When confronted with a decision that involves a fairly large amount of information (such as a medical, political, or legal decision), it is reasonable to assume that a decision maker will draw on his or her memory for information about the decision in some manner (Hastie \& Park, 1986). Research on the decision process, however, has spawned differing views on the role that memory plays in these types of decisions. An availability view asserts that the amount of information available in memory that favors different options will be the primary determinant of the decision (Reyes, Thompson, \& Bower, 1980). Other views put more emphasis on the structure of the memory representation of the decision maker. The explanationbased decision-making theory asserts that an overall picture of the information is relied on in the decision process, including inferences and ideas about how the world works. Pennington and Hastie (1986, 1988, 1993) have argued that a causal model of decision information is used in decision situations in which a large amount of information is relevant, especially when some of the information may be incomplete or even contradictory. Clarifying the distinction between decisions based on memory of actual information and a causal interpretation of decisionrelevant information will expand current knowledge about the role of memory in decision making.

Support for this research was provided by NSF Grant SES-9113479 to the second author. We gratefully acknowledge the assistance of Michelle Owens in the collection and coding of the data. We also thank Susan Goldman, Sashank Varma, and two anonymous reviewers for helpful comments. Correspondence concerning this article should be addressed to M. B. W. Wolfe, Learning Technology Center, Vanderbilt University, Box 45 GPC, Nashville, TN 37203-0045 (e-mail: mike. wolfe $($ vanderbilt.edu).
In discussing the relationship between memory and decisions, Hastie and Park (1986) distinguished between judgments that are made on line and memory-based judgments. On-line judgments are those in which a decision is made concurrently with the presentation of the evidence. Each piece of evidence is used to update or revise a current judgment. Memory-based judgments refer to those in which a person is required to retrieve information from memory to make a decision or judgment that was not made at the time of the encoding of the information. The juror decision task is typically thought to be a memory-based decision task, because jurors retrieve information from memory during the process of arriving at a verdict. Hastie and Pennington (1989) further distinguish between direct memory-based judgments, which are based on memory of direct information, and inferencememory-based judgments, in which decisions are made on the basis of inferences made at an earlier time that are recalled at the time of the decision. The availability model represents a direct memory-based theory, whereas the explanation-based decision-making theory is an example of an inference-memory-based theory. The goal of the present research is to distinguish between these two theoretical explanations of the role of memory in judgment.

\section{AVAILABILITY}

An availability model of judgment is an example of a model in which a direct relationship between memory and judgment is proposed, as is shown in Figure 1. In this model, information pertaining to a decision is presented to the decision maker in the form of evidence favoring Decision A and evidence favoring Decision B. No specific claim is made about how the information is organized in memory, however. The decision process involves 


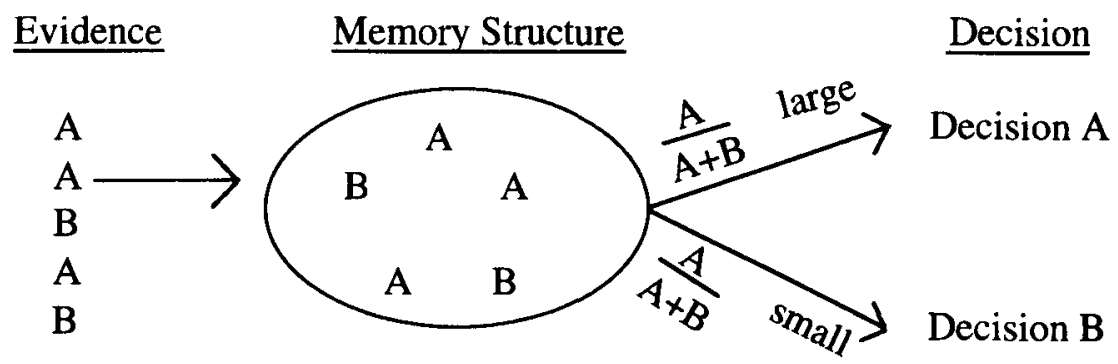

Figure 1. The availability model of decision making.

a sampling of evidence favoring Decisions $A$ and $B$, with the critical factor being the relative amount of evidence recalled favoring each decision. If the proportion of evidence recalled that favors Decision $\mathrm{A}$, relative to the total amount of evidence recalled, is large, Decision A will tend to be chosen. If the proportion of evidence recalled that favors Decision A, relative to the total amount of evidence recalled, is small, Decision $B$ will tend to be chosen.

The availability model has been used to explain decision-making behavior in certain situations. Tversky and Kahneman (1973) have argued that subjects use an availability heuristic in making judgments of frequency and probability. Reyes et al. (1980) have argued that the availability strategy can also be used to describe subjects' behavior in a juror decision task after a delay between evidence presentation and the decision. The subjects in the Reyes et al. study read evidence statements in a mock juror situation. The memorability of the evidence was manipulated by adding vivid but unimportant phrases to either prosecution or defense evidence. Judgments of guilt and free recall of the evidence statements were collected both immediately after the statements were read and after a 48-h delay. After the delay, there was a significant correlation between memory for the evidence and the subjects' judgments of guilt. Those subjects who recalled more prosecution evidence, relative to defense evidence, gave higher ratings of guilt. Reyes et al. interpreted these results as evidence that after a delay, the subjects based their judgments on the information that was available in memory at the time of the judgment.
An alternative version of the availability model, also examined by Reyes et al. (1980), is one in which the evidence recalled by a person is weighted by its importance in determining what impact it will have on the judgment. In addition to memory and decisions, Reyes et al. also collected ratings of the importance of each piece of evidence. Their results showed that when evidence that was recalled was weighted by its importance, the correlation between memory for evidence and judgment increased from .31 to .60 . In both cases, however, no claim was made about the structure of the evidence in memory.

\section{EXPLANATION-BASED DECISION MAKING}

Pennington and Hastie $(1986,1993)$ have argued that an explanation-based decision-making theory accounts for such decisions as a juror's weighing evidence in a trial (Figure 2). The explanation-based decision-making theory is an example of an inference-memory-based theory, in which a memory structure is created on the basis of information presented. The crucial factor in explanation-based decision making is that the decision maker imposes order on the information presented. Inferences are formed to help make sense of the information, and an organized memory structure is constructed. Research on text comprehension has shown that readers spontaneously utilize their prior knowledge in comprehending texts (Kintsch, 1988) and that they spontaneously generate inferences to fill in gaps in texts (Graesser, Singer, \& Trabasso, 1994). In the decision process, the

\section{Evidence $\quad$ Memory Structure $\quad$ Decision}

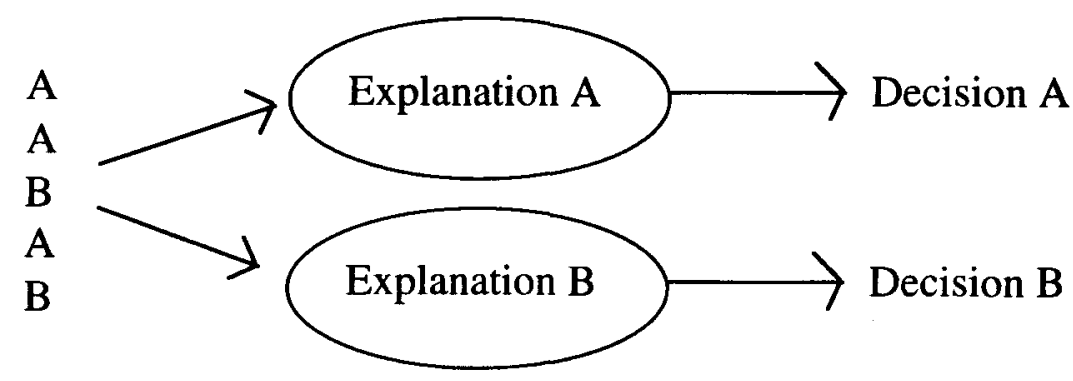

Figure 2. The explanation-based model of decision-making. 
memory structure is compared with each of the potential decision options, and the option that more closely matches the memory structure is more likely to be chosen.

According to explanation-based decision making, then, the decision is not based on memory for the literal information presented but, rather, on a causal representation of the trial information that is constructed by the juror upon hearing the testimony (Pennington \& Hastie, $1986,1988,1992)$. This causal representation is assumed to take the form of a story of "what happened" that is constructed on the basis of both actual testimony and inferences generated in response to the testimony. This assertion, that the memory structure is in the form of a story, has led to the theory's being called the story model. For example, Pennington and Hastie (1986) analyzed verbal protocol data from subjects who had watched a video of a mock trial. Protocol statements revealed that memory for trial information was organized like stories, with structured episodes and causal chains of events linking episodes together. In addition, differences in the subjects' representations of the information correlated with differences in their verdict decisions. Other decisionmaking theories also claim that some sort of intermediate mental model is constructed, using inferences generated on the basis of the actual information presented (Beach, 1993; Klein, 1993; Kuhn, Weinstock, \& Flaton, 1994).

\section{DISTINGUISHING BETWEEN THEORIES}

In a juror decision experiment, Pennington and Hastie (1988) presented evidence for memory-based decisions mediated by a juror's representation of the trial evidence by showing that verdict choices are affected by the relative coherence with which prosecution evidence and defense evidence are presented. Testimony from each witness addressed events at a particular point in the time sequence of the events described. Some witnesses testified about events that occurred at a particular time before the crime, and other witnesses testified about events that either were directly related to the crime or happened after the crime. The coherence of the testimony was manipulated separately for the prosecution and the defense by altering both the presentation order of the witnesses and the order of the testimony within each witness. In the coherent version of testimony, the order of witness testimony was such that events were revealed in testimony in the temporal order in which they happened. In a version more like an actual trial, the temporal ordering of the events was disrupted by altering the order of the witnesses and the order of testimony presented within each witness. The evidence order manipulation was presumed to represent a difference in the coherence of the evidence and, thus, a difference in the ease with which a story of the events could be constructed by the subjects. When the prosecution evidence was presented in the coherent order, the subjects were more likely to return a guilty verdict than when the prosecution was presented in a manner more consistent with how trial information is typically presented. Conversely, if the defense evidence was presented in the coherent order, the subjects were more likely to find the defendant not guilty.

The availability model could also account for these results by proposing that the greater coherence in the temporally ordered version would likely result in those items being more available in memory than were the items in the less coherent version. Research on narrative comprehension suggests that information will be better remembered if it is presented in a coherent fashion (Kintsch, Mandel, \& Kozminsky, 1977; Thorndyke, 1977). For example, Thorndyke found that recall was increased with greater story coherence (as judged by the amount of plot structure in a story). According to the availability model, then, decisions in the Pennington and Hastie (1988) study would favor the temporally ordered version, because more information that favored it would be available in memory. Pennington and Hastie (1988) did not assess the availability of the evidence in memory, however, so these two theoretical accounts cannot be distinguished on the basis of their data.

In the present experiments, we examined the relationship between evidence coherence, memory for evidence, and verdicts. Evidence coherence was manipulated by altering the order of witness testimony and the order of testimony within each witness. We presumed that presenting prosecution and defense evidence in a coherent fashion would facilitate the process of constructing an explanatory representation of the events being described (a story). Thus, we predicted that for prosecution and defense evidence, verdicts would favor the more coherent presentation orders. When the prosecution was presented in a coherent order, subjects would be more likely to find the defendant guilty, and when the defense was presented in a coherent order, subjects would be more likely to find the defendant not guilty. We also predicted that when evidence was presented in a more coherent fashion, availability of the evidence in memory would be greater.

The ability of both explanation-based and availability theories to account for the predicted relationship between memory and judgment represents a general issue in distinguishing between these theories. Factors that tend to make it easier for subjects to construct causal structures of decision information also tend to make that information more readily available in memory. In these experiments, we utilized two methods to distinguish between these theoretical accounts. First, the ability of evidence coherence and availability to account for decisions could be assessed by examining the effects of each variable on decisions while statistically factoring out the other (Shedler \& Manis, 1986). The evidence order manipulation and amount of recall could be used as multiple predictors in regression models to predict subjects' decisions. One caveat to this method is that in these experiments, evidence presentation order was manipulated, whereas recall would vary naturally between subjects. As a result, it was important to establish that there was a sufficient amount of variability among the recall scores so that both theories had the potential to account for variability in decisions. The other method for distinguishing evidence 
coherence from memory for evidence was experimental. If case materials could be constructed in which evidence coherence varied across conditions but the memorability of the evidence did not, decisions could be examined under circumstances in which memorability for evidence could not account for decisions.

\section{EVALUATING THE COHERENCE OF THE EVIDENCE}

In Experiments 1 and 2, we directly examined the validity of the evidence presentation order manipulation by measuring the coherence of each of the presentation order conditions. In the Pennington and Hastie (1988) study, coherence was manipulated by ordering the presentation of evidence so that the causal and temporal relationships among the events described either were preserved in the evidence presentation order or were not preserved. It was presumed that this evidence order manipulation constituted a difference in the coherence of the evidence across the conditions, which resulted in differences in the ease with which subjects could construct stories explaining the evidence. Ample evidence exists that comprehension of texts is facilitated by coherence, as compared with texts that are less coherent (Bower, Black, \& Turner, 1979; Britton \& Gülgöz, 1991; Kintsch et al., 1977; McNamara, Kintsch, Songer, \& Kintsch, 1996). For example, Britton and Gülgöz (1991) improved the coherence of textbook passages by creating more common reference words between sentences in the passages. Recall of the text information was greater for the more coherent passages. Kintsch et al. (1977) had subjects read stories that either were in their natural order or had the paragraphs scrambled. Reading times were longer for the scrambled stories, suggesting that processing of the stories was more difficult. These results suggest that the temporally ordered version of the trial evidence in the Pennington and Hastie (1988) study was, in fact, more coherent, thereby making it easier to construct a story than it would be when the temporal order of events was disrupted. In this study, however, we wished to directly assess the coherence of the evidence presentation conditions, to ensure that the evidence order manipulation did result in coherence differences. We assessed the coherence of the texts by using a new computational model called latent semantic analysis (LSA), which has been shown to provide reliable assessments of text coherence.

The LSA model of knowledge representation (Landauer \& Dumais, 1997; Landauer, Foltz, \& Laham, 1998) has been utilized by Foltz, Kintsch, and Landauer (1998) as a method for determining the coherence of a text passage. LSA is a statistical method for approximating the semantic associations readers acquire between words and text passages. ${ }^{1}$ The coherence of a text is defined as the average semantic similarity (on a scale from -1 to +1 ) between sentence $n$ and sentence $n+1$ across the entire text. These LSA coherence scores correlated extremely highly with free-recall data and answers to questions about several texts. We adopted this method of assessing co- herence to evaluate the coherence differences between the two evidence presentation orders. If the temporal ordering of evidence does indeed result in greater coherence, the average similarity of adjacent evidence items to each other should be higher than in the condition in which temporal ordering is not preserved.

\section{EXPERIMENT 1}

In the first experiment, we attempted to replicate Pennington and Hastie (1988) by providing further evidence that coherence manipulates decisions, using a new stimulus trial. Testimony from each prosecution and defense witness addressed events related to a particular point in the timeline of events related to the trial. For both prosecution and defense, the coherence of the evidence was manipulated by altering the order in which witnesses were presented and the order of testimony within each witness. In the temporal (or story) order, testimony was ordered in such a way that the events described were revealed in the order in which they happened. In witness order, each witness presented the same testimony as that in the story order, but the order within and between witnesses was changed, so that the temporal nature of the testimony was disrupted, much like an actual trial. The subjects made decisions about the guilt of the defendant and provided free recall of the trial evidence. We predicted that both decisions and memory for evidence would favor the more coherent versions of prosecution and defense evidence.

The legal case used in this experiment had one important difference from the case used in Pennington and Hastie (1988). Pennington and Hastie (1988) used a case in which the defense strategy was to present evidence that directly addressed the points made by the prosecution. In this way, both prosecution and defense testimony addressed a common set of events, with critical discrepancies regarding some particularly important events. In the present experiment, a defense strategy was to present a plausible but different explanation for the crime (that another person other than the defendant could be guilty). Presumably, if the story presented by the defense was plausible, there would be doubt about the prosecution story, and the defendant would be found not guilty. Both strategies are plausible legal strategies that could be used in an actual trial.

Experiment 1 had several purposes. First, we wished to replicate Pennington and Hastie (1988), using different materials, and establish that both explanation-based and availability theories could account for our results. We also examined a weighted availability model, in which recalled evidence items were weighted by their importance. Because Pennington and Hastie (1988) did not measure memory for trial information, the ability of both theories to account for the predicted relationship between memory and decisions has not been established. Second, the relationship between memory and decisions was to be examined statistically. In multiple regression analyses, evidence presentation order and recall was used to pre- 
dict decisions. These analyses determined the effect of each variable on decisions after factoring out the effects of the other. Importantly, these analyses were not hindered by the predicted result that presentation order and recall would both be independently predictive of decisions. The final purpose of Experiment 1 was to inform us about the memory differences in specific evidence statements as a function of the presentation order manipulation. In Experiment 2, we attempted to equate memory for evidence as a function of evidence presentation order by increasing the memorability of certain evidence items. The results of Experiment 1 informed the decisions about the specific manipulations that would be made.

\section{Method}

Materials. A stimulus trial was constructed for this Experiment that we called State of Texas vs. Ellen Masters (based on materials developed by Schum \& Martin, 1982). In this trial, the defendant, Ellen Masters, was accused of malicious destruction. A convertible Mercedes belonging to Tammy Clark had been set on fire late at night in the carport where Clark lived. The prosecution claimed that Masters' husband was having an affair with Clark and that Masters burned Clark's car in revenge. The defense suggested that Clark's ex-fiancée, Thomas Lloyd, could have been responsible for burning the Mercedes in anger over a broken engagement.

The stimulus trial began with an indictment, followed by a brief prosecution opening statement. Eight prosecution witnesses then presented a total of 86 statements. A brief defense opening statement was then followed by the testimony of eight defense witnesses, who presented a total of 77 statements. The judge's instructions to the jury gave a definition of malicious destruction and explained that the burden of proof was on the prosecution. The instructions stated that if the plaintiff had convinced the subject that the defendant was guilty, a guilty verdict must be returned. The trial was presented as a 20-page booklet with a single, new page for the indictment, for each opening statement, for each new witness, and for the judge's instructions.

Two orders of both the prosecution and the defense evidence were devised. In the story order, witness testimony was ordered so that the trial information was revealed in the temporal and causal order of the events described. This meant that the first witnesses presented information about the nature of the disagreement between the plaintiff and the defendant and about the events leading up to the crime. Later witnesses testified about the actual crime and about evidence found at the crime scene. As much as possible, this ordering was preserved both within and across witness testimony. In the witness order, the natural temporal and causal order of events was disrupted. This was accomplished by altering both the ordering of witnesses and the ordering of testimony within witnesses. In many ways, the witness order resembled a presentation order more natural in trials. That is, evidence is first presented that a crime occurred. Evidence about the events leading up to the crime is often presented later. In both presentation orders, each witness presented the same information; only the order was altered.

The two prosecution (story/witness) and two defense (story/ witness) orders were combined to create four different versions of the case. In one version, both sides were presented in story order, whereas another version had both sides in witness order. A third version had the prosecution in story order and the defense in witness order, and the fourth version had the prosecution in witness order and the defense in story order. In all cases, the prosecution was presented first, since this is the pattern in an actual trial.

To ensure that order manipulation effects were not due to primacy or recency of "important" information, a separate group of 40 subjects ( 10 in each of the four versions) read the evidence and rated the importance of each item to the prosecution or the defense case on a scale from 0 to 10 . Importance ratings had an overall mean of 5.2 and a standard deviation of 1.96 , suggesting reasonable variability in the ratings. Correlations were then calculated between the serial position of each item in the testimony and the mean importance rating of the item for the prosecution and the defense in each of the two evidence order conditions. Analysis of the correlations showed no significant difference between story and witness orders for either the prosecution or the defense, indicating that in both evidence sets, "important" information was distributed comparably across the serial positions for the two presentation order conditions.

To assess the effect of the evidence order manipulation on the coherence of the trial evidence, both the story and the witness versions of the evidence were submitted to Foltz et al.'s (1998) method of assessing coherence using LSA (described earlier). For both presentation orders, the semantic similarity was computed between the first and the second evidence statements, the second and the third statements, and so on to the end of the statements. The similarity scores for each of the presentation orders were then evaluated. For the prosecution evidence, the overall coherence was higher for the story order (mean $=.36)$ than for the witness order [mean $=.21$; $F(1,168)=21.48, p<.0001]$. For the defense evidence, the story order was also more coherent (mean $=.36$ ) than the witness order $[$ mean $=.26 ; F(1,152)=8.15, p=.005]$. Thus, the coherence was successfully manipulated by altering the temporal ordering of the evidence.

Subjects. Sixty undergraduates from the University of Colorado served as subjects. The subjects received course credit for their participation. The subjects were randomly assigned to one of the four evidence order conditions.

Procedure. The subjects were run in groups of 1 to 5 in a small room, with dividers between the subjects. On arrival, they were told that the Experiment was concerned with juror decision making. An instruction sheet read aloud by the experimenter explained that the subjects would read evidence from a trial and then would be asked to make a decision and answer some questions. The subjects read the stimulus trial one page at a time. They were not allowed to turn back to previous pages after progressing to the next page.

After reading the trial, the subjects responded to several kinds of questions. First, they made a verdict choice of guilty or not guilty of malicious destruction. The verdict choice did not specifically mention beyond a reasonable doubt as a criterion. Confidence in the verdict choice was then assessed by a rating on an 11-point scale $(0=$ not at all confident, $10=$ very confident $)$. Evidence strength ratings for the prosecution and the defense were also measured on an 11-point scale $(0=$ evidence is very weak, $10=$ evidence is very strong). The subjects rated likelihood of guilt on a 21-point scale $(-10=$ Masters is definitely innocent, $+10=$ Masters is definitely guilty). Finally, the subjects recalled from memory as many of the 163 evidence statements as they could and wrote them in the spaces provided. No recall cues or instructions about recall order were provided. Exact wording did not need to be recalled, but the subjects were asked to be as close to the original wording as they could.

\section{Results}

Decisions. We expected the ordering of evidence to affect decisions, with the decisions favoring evidence that was presented in the temporal order that corresponded to the events described (story order). These results would be consistent with both the explanation-based and the availability models. To test our hypothesis, a log linear model was fit to the verdict frequency data (see Table 1). As we predicted, the subjects were more likely to decide that the defendant was guilty of malicious destruction when the prosecution was presented in story order than when it was presented in witness order $\left[\chi^{2}(1, N=60)=\right.$ $4.45, p=.035]$. Contrary to our hypothesis, however, pre- 
Table 1

Percentage of Subjects Choosing a

Verdict of Guilty of Malicious Destruction

by Prosecution and Defense Order Conditions

\begin{tabular}{lccc}
\hline & \multicolumn{3}{c}{ Prosecution Evidence } \\
\cline { 2 - 4 } Defense Evidence & Story Order & Witness Order & Mean \\
\hline Story order & 53.3 & 20.0 & 36.7 \\
Witness order & 46.7 & 26.7 & 36.7 \\
Mean & 50.0 & 23.4 & \\
\hline
\end{tabular}

senting the defense in story order versus witness order had no effect on subjects' judgments of guilt. There also was no interaction $\left(\chi^{2}<1\right)$.

The verdict results for the prosecution condition were confirmed by results from the subjects' ratings of the likelihood of guilt. When the prosecution was presented in story order, the subjects rated the defendant as more likely to be guilty (with a mean of 3.77 on a scale from -10 to 10 ) than when it was in witness order [with a mean of $-0.45 ; F(1,56)=15.59, p=.0002]$. Also consistent with the verdict results, the defense order manipulation did not significantly affect likelihood of guilt ratings; nor was there an interaction $(F \mathbf{S}<1)$.

We also predicted that the evidence order manipulation would affect ratings of evidence strength and confidence in the verdict (see Table 2). For the prosecution, evidence strength ratings were higher when the evidence was presented in story order than when it was not $[F(1,56)=$ $7.20, p=.01]$. In addition, prosecution condition affected ratings of defense strength so that when prosecution evidence was presented in story order, ratings of defense strength were lower $[F(1,56)=4.63, p=.04]$. Again consistent with verdict results, neither ratings of prosecution strength nor those of defense strength were affected by the defense order manipulation, and no interactions were present. Our prediction that confidence in the chosen verdict would be greater if the subject saw the evidence in story order was not supported.

Memory. For the recall analysis, witness testimony was first broken into idea units. Each idea unit consisted of a single semantic unit presented as witness testimony. For example, the statement "There was a car fire" would spawn the idea units "There was a fire," and "A car was on fire." This allowed a subject to be given credit for recalling part of a statement without remembering the entire statement. Two raters independently coded each recall protocol with approximately $90 \%$ agreement. Disagreements were resolved by discussion. Incorrectly recalled statements and inferences were not included in the analysis. Idea units that were repeated within a subject were counted only once.

We predicted that subjects who received evidence in story order would have greater recall for that evidence than would subjects who saw the same evidence in witness order. Again, such results would be consistent with both explanation-based and availability theories. Out of 311 idea units ( 183 for the prosecution and 129 for the defense), the subjects recalled an average of approximately 77 idea units ( 49 prosecution units and 28 defense units).
The recall measure used was the percentage of prosecution evidence recalled, relative to the total evidence recalled for each subject. This measure was used for the analyses because the availability hypothesis states that the crucial factor in a decision is the relative amount of information recalled favoring each side. Across conditions, $63 \%$ of the evidence recalled was prosecution evidence. A general linear model analysis was conducted to test for all main effects and interactions. As is shown in Table 3, the percentage of prosecution evidence recalled, relative to total recall, was greater when the prosecution was presented in story order than when it was presented in witness order $[F(1,56)=4.52, p=.04]$. Similarly, the percentage of evidence recalled that was prosecution evidence was less when the defense was in story order than when it was in witness order $[F(1,56)=10.83, p=.002]$. The interaction was marginally significant $[F(1,56)=$ $3.26, p=.08]$.

Memory for evidence was also examined as a weighted function of the importance of the evidence. The importance ratings collected by a separate group of subjects (on a scale from 0 to 10 ) were used in these analyses. Recall scores for each evidence item were calculated by using the importance rating for that item, rather than by counting all evidence items equally. The percentage of prosecution evidence as a function of total evidence recalled was then calculated as before. As with the unweighted data, the percentage of prosecution evidence relative to total evidence recalled was higher for the subjects who received prosecution evidence in story order (mean $=$ $64.1 \%$ ) than for those who received prosecution evidence in witness order $[$ mean $=59.6 \% ; F(1,56)=8.49$, $p=.005]$. Also as with the unweighted data, the subjects who received defense evidence in story order recalled a smaller percentage of prosecution evidence (mean $=$ $58.6 \%$ ) than did those who received defense evidence in witness order $[$ mean $=65.1 \% ; F(1,56)=17.79, p<$ $.0001]$, and there was no interaction.

In addition to examining evidence order condition and memory as separate predictors of decisions, we examined both simultaneously in multiple regression analyses. For these analyses, we used the likelihood of guilt ratings instead of verdicts because they were on a continuous

Table 2

Mean Ratings of Strength of Prosecution and Defense Evidence by Prosecution and Defense Order Conditions

\begin{tabular}{|c|c|c|c|}
\hline \multirow[b]{2}{*}{ Defense Evidence } & \multicolumn{3}{|c|}{ Prosecution Evidence } \\
\hline & Story Order & Witness Order & Mean \\
\hline \multicolumn{4}{|c|}{ Ratings of Prosecution Evidence Strength } \\
\hline Story order & 6.1 & 4.1 & 5.1 \\
\hline Witness order & 5.7 & 4.8 & 5.3 \\
\hline Mean & 5.9 & 4.4 & \\
\hline \multicolumn{4}{|c|}{ Ratings of Defense Evidence Strength } \\
\hline Story order & 3.7 & 4.5 & 4.1 \\
\hline Witness order & 3.7 & 4.8 & 4.3 \\
\hline Mean & 3.7 & 4.7 & \\
\hline
\end{tabular}

Note--Strength ratings range from 0 (evidence is very weak) to 10 (evidence is very strong). 
Table 3

Percentage of Prosecution Evidence Recalled as a Function of Prosecution Condition and Defense Condition

\begin{tabular}{lccc}
\hline & \multicolumn{3}{c}{ Prosecution Evidence } \\
\cline { 2 - 4 } Defense Evidence & Story Order & Witness Order & Mean \\
\hline Story order & 61.0 & 60.5 & 60.7 \\
Witness order & 69.2 & 62.9 & 66.1 \\
Mean & 65.1 & 61.7 & \\
\hline
\end{tabular}

scale and, therefore, should represent a more sensitive measure of a subject's belief in the guilt of the defendant. By using both evidence order condition and recall of information to predict likelihood of guilt ratings, the influence of each measure could be examined while statistically factoring out the other. Since recall of information was a dependent variable and was not specifically manipulated, it was also necessary to establish that there was sufficient variability in the recall scores to warrant these multiple regression analyses. The percentage of prosecution evidence recalled relative to total recall had a standard deviation of $7 \%$. Thus, there was sufficient variability in the recall scores to potentially account for differences in likelihood of guilt.

Simple correlations between recall and likelihood of guilt were calculated in addition to the multiple regression analyses (simple effects of presentation order condition on likelihood-of-guilt ratings were presented earlier). The percentage of prosecution evidence recalled, relative to total evidence recalled, was marginally correlated with likelihood of guilt $[r(58)=.25, p=.05]$. For the multiple regression analyses that included recall and prosecution condition, prosecution condition was a reliable predictor of likelihood-of-guilt ratings over and above the effects of recall $[F(1,57)=12.84, p=.0007]$. Recall, however, was not a reliable predictor of likelihood-ofguilt ratings over and above evidence order condition $[F(1,57)=1.52]$. For the multiple regressions that included defense condition and recall, evidence order condition was again not a reliable predictor of likelihood of guilt $(F<1)$, whereas recall was a marginal predictor of likelihood of guilt $[F(1,57)=3.31, p=.07]$. Thus, recall and subjects' ratings of the likelihood of guilt were correlated, but when prosecution condition was included in a multiple regression, recall no longer predicted likelihood of guilt. When defense condition was added in a multiple regression, recall remained a predictor.

For the recall weighted by importance of evidence, recall was also correlated with likelihood of guilt $[r(58)=$ $.28, p=.03]$. The multiple regression results for the prosecution condition were qualitatively the same as those for the unweighted results. For the prosecution, evidence order condition was a reliable predictor of likelihood of guilt over and above recall $[F(1,57)=11.87, p=.001]$, whereas recall was not a reliable predictor of likelihood of guilt over and above evidence order condition $[F(1,57)=$ $1.53]$. For the defense, evidence order condition was not a predictor over and above recall $(F<1)$, but again recall was a reliable predictor over and above evidence order condition $[F(1,57)=4.23, p=.04)$.

\section{Discussion}

When prosecution stories were presented coherently, the subjects were more likely to find the defendant guilty than when prosecution stories were less coherent, partially replicating Pennington and Hastie (1988). We presume that the coherent version of the prosecution evidence made it easier for the subjects to construct a story explaining the events than it was when the evidence was less coherent. These results did not hold for the defense, however. The subjects were equally likely to find the defendant guilty whether the defense story was coherent or not. The coherent prosecution story also elicited greater ratings of likelihood of guilt and prosecution evidence strength. As was also predicted, the percentage of prosecution evidence recalled, relative to total recall, was significantly greater for the more coherent prosecution order than for the less coherent order.

These results are consistent with both the story model and the availability model. The story model accounts for the prosecution results, because the verdicts favored the evidence presentation condition, which presumably made it easier for the subjects to construct a story consistent with the prosecution evidence. The availability model accounts for the same results, because the subjects who received prosecution evidence in the coherent order recalled more relative prosecution evidence, which could be responsible for the verdict results. Thus, it is established that for the basic decision and memory results, both theories in question are able to account for the prosecution results.

Multiple regression results in which evidence presentation order and memory for evidence were used to predict likelihood of guilt were consistent with the story model for the prosecution. The evidence order condition was predictive of likelihood-of-guilt ratings over and above the amount of evidence recalled, with the coherent story being correlated with higher likelihood-of-guilt ratings. Recall was not predictive of likelihood of guilt over and above the evidence order condition. For the defense, however, recall was predictive of likelihood-of-guilt ratings over and above the evidence order condition, with lower relative prosecution recall being correlated with lower likelihood-of-guilt ratings.

The failure to replicate the role of defense stories in verdict decisions introduces a potential boundary on Pennington and Hastie's story model for juror decisions. Pennington and Hastie (1988) found verdicts favoring the temporally ordered evidence for both the prosecution and the defense. Because we did not find defense effects, there is a question of what happened with the defense story and what implications it might have for the story model. One reason for the failure may be that story construction is not as important for the defense as it is for the prosecution (Pennington \& Hastie, 1988), because the burden of proof is on the prosecution. Thus, it may be more important for the prosecution to provide a plausible account of the events than it is for the defense. Another possibility is that the defense tactic in this case was to introduce an alternate defendant (a previous boyfriend). 
The story that was presented had to do with the events in their relationship leading to the breakup and subsequent bad feelings between the ex-boyfriend and the victim. Thus, the details of the story provided by the defense strengthened the theme that someone else could have done it but did not go beyond that theme to provide an account of exactly how the alternate offender actually committed the crime. The defense also did not attempt to directly discredit prosecution evidence. Thus, it is possible that a story that merely strengthens a theme will not affect decisions in the same way in which one that provides a true alternate account would do. In previous work, Pennington and Hastie (1988) used a defense case that directly addressed the claims being made by the prosecution.

Because the questions regarding the lack of verdict effects in the defense case were not related to the issue of discriminating between explanation-based and availability accounts of the role of memory in decisions, they were not addressed in Experiment 2. Furthermore, because of the lack of verdict effects as a function of defense evidence presentation order, it was sufficient to examine only the relationship between memory and decisions for the prosecution case in Experiment 2.

\section{EXPERIMENT 2}

In Experiment 2, we attempted to provide experimental evidence that verdicts are affected by prosecution evidence coherence even when memory for evidence is not affected by evidence coherence. We accomplished this goal by eliminating the relative memory differences that were a function of prosecution coherence found in Experiment 1 . Verdicts and likelihood-of-guilt ratings could then be examined under a situation in which the results could not be attributed to differential memory for evidence. We predicted that even in a situation in which the more coherent and less coherent orders showed no memory difference, decisions would still favor the more coherent prosecution story.

\section{Method}

Materials. For Experiment 2, the same case of State of Texas vs. Ellen Masters was used. In order to eliminate memory differences between the prosecution story and witness orders, certain evidence items were repeated when the prosecution was presented in witness order. An item-by-item analysis of recall from Experiment 1 showed 25 idea units that had recall probability differences of at least $10 \%$, with these particular items being more likely to be recalled if the prosecution evidence was presented in story order, rather than in witness order. Each of these particular idea units was repeated twice within the prosecution witness who had originally stated the idea unit. The importance ratings of the idea units (from Experiment 1) that were repeated had a mean of 6.29. This was greater than the overall mean importance rating of 5.2 given to the items in Experiment 1 . Thus, the results cannot be attributed to memory's being equated by the repetition of irrelevant information.

Repetition of idea units was achieved by changing the statement in which the idea unit was originally stated, but without adding any new relevant information. For example, the statement "Once, Ellen Masters accused me of having an affair with her husband" had a greater probability of being remembered by the subjects who received prosecution evidence in story order than by those who received prosecution evidence in witness order. In order to repeat this idea unit, the statement "It was right after aerobics class that Ellen Masters accused me of having an affair with her husband" was added within the same witness. The spacing effect was utilized in order to maximize the effectiveness of the repetitions on memory (Melton, 1970). Repetition statements were positioned within each witness in such a manner that as many items as possible were positioned between the original statement and the repetitions. Aside from the repetitions of the 25 idea units, all materials, including the defense case, were identical to those in Experiment 1.

As in Experiment 1, we examined the coherence of the story and witness orders, using LSA. Since only the prosecution evidence in witness order was altered from Experiment 1, only the prosecution evidence was reexamined. As in Experiment 1, the prosecution story order was more coherent as measured by the LSA coherence measure $($ mean $=.36)$ than was the witness order $[$ mean $=.22 ; F(1,196)=$ $21.92, p<.0001]$.

Subjects. Sixty undergraduates from the University of Colorado served as subjects. The subjects received course credit for their participation. The subjects were randomly assigned to one of the four evidence order conditions.

Procedure. The procedure was identical to that in Experiment 1.

\section{Results}

Decisions. As in Experiment 1, we expected more subjects to choose guilty verdicts when the prosecution evidence was presented in story order than when it was presented in witness order. Table 4 shows the percentages of subjects choosing guilty verdicts as a function of prosecution and defense evidence order condition. As was predicted, and consistent with the results of Experiment 1, the subjects were more likely to decide that the defendant was guilty of malicious destruction when the prosecution was presented in story order than when it was presented in witness order $\left[\chi^{2}(1, N=60)=4.38, p=.04\right]$. Also as in Experiment 1, there were no significant differences in verdict choices owing to defense condition $\left[\chi^{2}(1\right.$, $N=60)=2.59]$ and no interaction $\left[\chi^{2}(1, N=60)=1.2\right]$. Notice in Table 4, however, that the trend in verdicts for the defense is in the opposite direction from that predicted. If anything, the subjects who received defense evidence in story order were more likely to find the defendant guilty.

Likelihood-of-guilt ratings were also higher for those subjects reading prosecution evidence in story order. The subjects rated the defendant as more likely to be guilty if they received prosecution evidence in story order (with a mean of 4.25 on a scale from -10 to 10 ), as compared with witness order [with a mean of $1.1 ; F(1,56)=6.00$, $p=.018]$. There were no significant effects of defense order condition on likelihood of guilt [means of 3.33 for

Table 4

Percentage of Subjects Choosing a Verdict of Guilty of Malicious Destruction by Prosecution and Defense Order Conditions

\begin{tabular}{lccc}
\hline & \multicolumn{3}{c}{ Prosecution Evidence } \\
\cline { 2 - 4 } Defense Evidence & Story Order & Witness Order & Mean \\
\hline Story order & 80.0 & 40.0 & 60.0 \\
Witness order & 46.7 & 33.3 & 40.0 \\
Mean & 63.3 & 36.7 & \\
\hline
\end{tabular}


story order and 2.02 for witness order; $F(1,56)=1.05$ ], and there was no interaction.

We also predicted that the evidence order manipulation would affect ratings of evidence strength (see Table 5). For the prosecution, evidence strength ratings were higher when prosecution evidence was presented in story order than when it was not $[F(1,56)=8.07, p=.006]$. The results from Experiment 1 showing that prosecution condition affected defense strength ratings were not duplicated in Experiment $2[F(1,56)=1.88]$. There were no effects of defense condition on prosecution or defense strength ratings and no interactions. Finally, as in Experiment 1 , there were no effects of confidence in verdicts as a function of prosecution or defense condition.

Memory. Recall of evidence was scored as the number of idea units recalled, as in Experiment 1. New idea units were added for the new information that accompanied the repetition statements (41 new idea units were added, for a total of 352 idea units). An average of 73 total idea units were recalled ( 48 prosecution units and 25 defense units). Three raters independently coded the freerecall data, achieving approximately $90 \%$ agreement, Disagreements were resolved by discussion.

We predicted that the repetition of prosecution statements for those who received prosecution evidence in witness order would eliminate the recall advantage for prosecution evidence presented in story order. As in Experiment 1 , the percentage of evidence recalled that was prosecution evidence was calculated and used as the unit of analysis. Across conditions, $66 \%$ of the total recall was prosecution evidence. A general linear model analysis was conducted to test for all main effects and interactions. As is shown in Table 6, there was no significant difference between the percentages of prosecution recall as a function of prosecution evidence order condition $[F(1,56)=$ 1.78]. Importantly, however, the mean recall percentage was greater for subjects in the witness order condition than for those in the story order condition. As a result, there is no question that the subjects who received prosecution evidence in story order did not recall relatively more prosecution evidence. In contrast to Experiment 1 , there was also no significant difference in recall as a

Table 5

Mean Ratings of Strength of Prosecution and Defense Evidence by Prosecution and Defense Order Conditions

\begin{tabular}{lccc}
\hline & \multicolumn{3}{c}{ Prosecution Evidence } \\
Defense Evidence & Story Order & Witness Order & Mean \\
\hline \multicolumn{4}{r}{ Ratings of Prosecution Evidence Strength } \\
Story order & 7.1 & 5.1 & \\
Witness order & 6.3 & 5.0 & 5.1 \\
Mean & 6.7 & 5.1 & \\
\multicolumn{4}{c}{ Ratings of Defense Evidence Strength } \\
Story order & 3.8 & 4.0 & 3.9 \\
Witness order & 4.1 & 5.2 & 4.7 \\
Mean & 3.9 & 4.6 & \\
\hline
\end{tabular}

Note- Strength ratings range from 0 (evidence is very weak) to 10 (evidence is very strong).
Table 6

Percentage of Prosecution Evidence Recalled as a Function of Prosecution Condition and Defense Condition

\begin{tabular}{cccc}
\hline & \multicolumn{3}{c}{ Prosecution Evidence } \\
\cline { 2 - 4 } Defense Evidence & Story Order & Witness Order & Mean \\
\hline Story order & 65.1 & 68.4 & 66.7 \\
Witness order & 64.1 & 65.6 & 64.8 \\
Mean & 64.6 & 67.0 & \\
\hline
\end{tabular}

function of defense condition $[F(1,56)=1.06]$, and there was no interaction.

Recall was also examined as a weighted function of the importance of evidence items, as in Experiment 1. For the prosecution condition, the percentage of prosecution evidence recalled was not different when the prosecution evidence was presented in story order (mean $=$ $64.6 \%$ ) than when it was presented in witness order [mean $=64.8 \% ; F(1,56)=.01]$. There was also no effect of defense condition on recall [means $=65.8$ for story order and 63.5 for witness order; $F(1,56)=1.70$ ]. Thus, when evidence is weighted by its importance, it again provides the same qualitative result as the unweighted evidence analysis.

As in Experiment 1, multiple regression analyses were conducted to examine the independent contribution of prosecution evidence order condition and recall of evidence on likelihood-of-guilt judgments. The percentage of prosecution evidence recalled had a standard deviation of .07 . Thus, there was essentially the same variability in recall scores as in Experiment 1, so the potential for recall to be predictive of likelihood of guilt was established. The simple correlation of recall with likelihood of guilt was not significant $[r(58)=.16$, n.s.]. In the multiple regression analyses using recall and the prosecution evidence order condition, the prosecution condition was again a reliable predictor of likelihood-of-guilt ratings over and above the effects of recall $[F(1,57)=7.70, p=$ $.008]$. Recall was a marginal predictor of likelihood-ofguilt ratings over and above prosecution condition $[F(1,57)=3.05, p=.09]$, with higher percentages of prosecution recall being correlated with marginally higher ratings of likelihood of guilt. For the multiple regressions that included defense evidence presentation order and recall, neither was a reliable predictor of likelihood of guilt $(F \mathrm{~s}<1)$.

For the multiple regressions in which recall was weighted by evidence importance, recall was marginally correlated with likelihood of guilt $[r(58)=.22, p=.09]$. Thus, unlike Experiment 1, evidence of a simple correlation between recall and judgments is weak. For the multiple regressions using weighted recall and prosecution condition, prosecution condition was a reliable predictor of likelihood of guilt $[F(1,57)=6.47, p=.01]$, whereas recall was a marginal predictor $[F(1,57)=3.47$, $p=.07]$. In the regressions using defense evidence order condition and recall, neither was predictive of likelihood of guilt $(F \mathbf{s}<1)$. 


\section{Discussion}

Verdict choices, likelihood of guilt, and prosecution strength ratings were again affected by the coherence of the prosecution evidence. These results are consistent with previous research supporting an explanation-based account of decision making (Pennington \& Hastie, 1986, 1988,1992 ). We presume that the more coherent evidence made it easier for the subjects to construct a representation of the evidence in the form of a story that explained the events. In addition, free-recall differences were eliminated as a possible account for the verdict effects; there was no difference in the relative amount of prosecution and defense evidence recalled as a function of prosecution condition. In fact, the relative amount of prosecution recall was slightly greater in the less coherent prosecution condition. There were also no differences in the recall of evidence weighted by its importance, ruling out a weighted availability model as an account of these data. Thus, an availability model of decision making, in which decisions are based on the relative amount of evidence recalled that favors each decision, cannot account for these decision results. Finally, assessments of the coherence of the evidence order conditions again established that the evidence presented in temporal order was more coherent than the evidence in which the temporal order was disrupted.

Further evidence supporting the story model is provided by the fact that in both Experiment 1 and Experiment 2, prosecution coherence was a reliable predictor of likelihood of guilt over and above the effects of recall on decisions. The ability of the recall measure to predict likelihood-of-guilt ratings over and above prosecution coherence was marginal and mixed across the two experiments. In Experiment 1, recall of defense evidence was a marginal predictor of likelihood of guilt, and in Experiment 2, prosecution recall was a marginal predictor of likelihood of guilt. These results are also consistent with the results of Shedler and Manis (1986), who found that recall of information did not predict judgments of the fitness of a parent after factoring out the vividness of the informational statements.

\section{GENERAL DISCUSSION}

These experiments provide evidence that the coherence of the information that explains events relevant to a decision is predictive of the decision, even when the relative amounts of information in memory favoring each side of the decision are equated. When the subjects were presented with prosecution evidence that was in the form of a coherent story, they were more likely to find the defendant guilty than when they were presented with prosecution evidence that was in a form more like that of a typical trial. The amount of trial information available in memory cannot account for these verdict results, because in Experiment 2, there was no significant difference in the amount of prosecution evidence recalled, relative to total evidence recalled, as a function of prosecution evidence coherence. Furthermore, multiple regression results in both experiments show that the coherence of prosecution evidence was a reliable predictor of likelihood-ofguilt ratings after factoring out the effects of memory for information on likelihood-of-guilt ratings. Conversely, memory for information was not a consistent predictor of likelihood-of-guilt ratings after factoring out the effects of prosecution coherence.

The coherence manipulation itself was also verified in these experiments. The LSA model of knowledge representation provided measurements of the coherence of the evidence presentation orders (Foltz et al., 1998). In both experiments, when the causal and temporal sequences of events in the world were preserved in the presentation of evidence, the evidence was more coherent (as assessed by LSA) than when the causal and temporal order was not preserved. These results provide evidence for the claim in the present study that the manipulation of evidence order did, in fact, constitute a manipulation of the ease with which a story corresponding to the evidence presented by each side of the case could be constructed.

The results of these experiments corroborate earlier work by Pennington and Hastie $(1986,1988)$ in providing evidence that an explanatory structure is a key intermediate mental state in the juror decision process. Earlier studies have shown that jurors' protocol data regarding trial information are in the form of stories, that inferences about the evidence are generated spontaneously, and that ease of story construction is an important factor in determining decisions (a result found in the present experiments as well). In concert with the present experiments, a strong case is made for the importance of explanations in decision situations in which decision makers are faced with large amounts of complex interrelated information.

Our primary hypotheses were tested by examining the effects of evidence coherence on verdicts and recall. However, an additional issue to be addressed is the possibility that the decision process itself causes a restructuring of memory (Dellarosa \& Bourne, 1984). Under this explanation, the decision process a person goes through would cause evidence in memory to be restructured in order to accommodate the verdict choice. According to this explanation, free recall of evidence for a person should favor the verdict choice made by the person. To test the possibility that the verdict process affects recall, verdict choices were used to predict recall. In neither Experiment 1 nor Experiment 2 did verdict choices predict recall. In other words, the subjects who chose a verdict of guilty did not remember a significantly higher percentage of prosecution evidence than did the subjects who chose not guilty. This evidence rules out a third potential relationship between memory and decisions for these experimentsthat the decision itself is responsible for differences in memory for evidence.

The generalizability of research supporting the story model has not yet been tested outside the legal decisionmaking domain. On the basis of this research and previous research supporting the story model, however, we make the claim that these results would generalize to situations in which decision makers are confronted with 
large amounts of complex interrelated information that could be put into a causal structure to explain the events related to the decision. Politics, economics, and medicine are examples of decision-making domains in which explanations of events relating to decisions could conceivably be constructed.

\section{REFERENCES}

BEACH, L. R. (1993). Image theory: Personal and organizational decisions. In G. A. Klein, J. Orasanu, R. Calderwood, \& C. E. Zsambok (Eds.), Decision making in action: Models and methods (pp. 148157). Norwood, NJ: Ablex.

Bower, G. H., BlaCk, J. B., \& Turner, T. J. (1979). Scripts in memory for text. Cognitive Psychology, 11, 177-220.

BrITTON, B. K., \& Gülgöz, S. (1991). Using Kintsch's computational model to improve instructional text: Effects of repairing inference calls on recall and cognitive structures. Journal of Educational Psychology, 83, 329-345.

Dellarosa, D., \& Bourne, L. E. (1984). Decisions and memory: Differential retrievability of consistent and contradictory evidence. Journal of Verbal Learning \& Verbal Behavior, 23, 669-682.

Foltz, P. W. KinTsCh, W., \& LANDAUeR, T. K. (1998). The measurement of textual coherence with latent semantic analysis. Discourse Processes, 25, 285-308.

Graesser, A. C., Singer, M., \& Trabasso, T. (1994). Constructing inferences during narrative text comprehension. Psychological Review, 101, 371-395.

Hastie, R., \& PARK, B. (1986). The relationship between memory and judgment depends on whether the judgment task is memory-based or on-line. Psychological Review, 93, 258-268.

Hastie, R., \& Pennington, N. (1989). Notes on the distinction between memory-based versus on-line judgments. In J. N. Bassili (Ed.), On-line cognition in person perception (pp. 1-17). Hillsdale, NJ: Erlbaum.

KINTSCH, W. (1988). The use of knowledge in discourse processing: A construction-integration model. Psychological Review, 95, 163-182.

KinTsCh, W., Mandel, T. S., \& KozMinsky, E. (1977). Summarizing scrambled stories. Memory \& Cognition, 5, 547-552.

KLEIN, G. A. (1993). A recognition-primed decision (RPD) model of rapid decision making. In G. A. Klein, J. Orasanu, R. Calderwood, \& C. E. Zsambok (Eds.), Decision making in action: Models and methods (pp. 138-147). Norwood, NJ: Ablex.

Kuhn, D., Weinstock, M., \& Flaton, R. (1994). How well do jurors reason? Competence dimensions of individual variation in a juror reasoning task. Psychological Science, 5, 289-296.

LANDAUER, T. K., \& DuMaIs, S. T. (1997). A solution to Plato's problem: The latent semantic analysis theory of acquisition, induction, and representation of knowledge. Psychological Review, 104, 211-240.

Landauer, T. K., Fol.TZ, P. W., \& Laham, D. (1998). An introduction to latent semantic analysis. Discourse Processes, 25, 259-284.
McNamara, D. S., Kintsch, E., Songer, N. B., \& KintsCh, W. (1996). Are good texts always better? Interactions of text coherence, background knowledge, and levels of understanding in learning from text. Cognition \& Instruction, 14, 1-43.

MeLton, A. W. (1970). The situation with respect to the spacing of repetitions and memory. Journal of Verbal Learning \& Verbal Behavior, 9, 596-606.

Pennington, N., \& Hastie, R. (1986). Evidence evaluation in complex decision making. Journal of Personality \& Social Psychology, 51, 242-258.

Pennington, N., \& Hastie, R. (1988). Explanation-based decision making: Effects of memory structure on judgment. Journal of Experimental Psychology: Learning, Memory, \& Cognition, 14, 1-12.

Pennington, N., \& Hastie, R. (1992). Explaining the evidence: Tests of the story model for juror decision making. Journal of Personality \& Social Psychology, 62, 189-206.

Pennington, N., \& Hastie, R. (1993). A theory of explanation-based decision making. In G. A. Klein, J. Orasanu, R. Calderwood, \& C. E. Zsambok (Eds.), Decision making in action: Models and methods (pp. 188-204). Norwood, NJ: Ablex.

Reyes, R. M., Thompson, W. C., \& Bower, G. H. (1980). Judgmental biases resulting from differing availabilities of arguments. Journal of Personality \& Social Psychology, 39, 2-12.

SCHUM, D. A., \& MARTIN, A. W. (1982). Formal and empirical research on cascaded inference in jurisprudence. Law \& Society Review, 17, $105-151$.

SHEDLER, J., \& MANIS, M. (1986). Can the availability heuristic explain vividness effects? Journal of Personality \& Social Psychology, 51, 26-36.

THORNDYKE, P. W. (1977). Cognitive structures in comprehension and memory of narrative discourse. Cognitive Psychology, 9, 77-1 10.

Tversky, A., \& KaHNEMAN, D. (1973). Availability: A heuristic for judging frequency and probability. Cognitive Psychology, 5, 207-232.

\section{NOTES}

1. An initial matrix that codes the occurrence of words in each of several thousand documents is submitted to a matrix decomposition procedure known as singular value decomposition. The dimensionality in which words and texts are represented is then reduced to about 300 , yielding a semantic space in which words and texts are represented as vectors in this high-dimensional space. The semantic similarity of words or texts can then be assessed by computing the similarity of the vector representations in this high-dimensional space (using the cosine between vectors as the measure of similarity; see Landauer \& Dumais, 1997, for details).

(Manuscript received December 15, 1997; revision accepted for publication August 13, 1999.) 